\title{
Formação do cirurgião em cirurgia laparoscópica do aparel ho digestivo. Experiência de 1.818 intervenções sem acidentes e sem mortalidade
}

\author{
H. W. Pinotti, C. E. Domene, P. Volpe, M. A. Santo, P. Onari
}

Trabalho realizado na Unidade de Cirurgia Laparoscópica da Disciplina de Cirurgia do Aparel ho Digestivo do H ospital das Clínicas da Faculdade de Medicina da Universidade de São Paulo, São Paulo, SP.

RESUMO - Desde a instalação da cirurgia laparoscópica no Hospital das Clínicas da Faculdade de Medicina da Universidade de São Paulo, a Disciplina de Cirurgia do Aparelho Digestivo passou a estruturar um programa para a formação do cirurgião em laparoscopia do aparelho digestivo.

Овјетіvo. A estrutura de ensino inclui a informação no ensino médico regular, extensão na Liga de Cirurgia Laparoscópica, formando o médico a partir de residência, principalmente em seu quarto ano, com estágio na Unidade de Cirurgia Laparoscópica de três meses.

MÉtodo. Este modelo de formação e preparo do cirurgião do aparelho digestivo assim implantado, com rigor, profundidade e seriedade é, certamente, responsável pelos resultados de nível de excelência obtidos.

Resultados. Exemplo disso representam as 1818 colecistectomias laparoscópicas realizadas na

\section{INTRODUÇÃO}

A Disciplina de Cirurgia do Aparel ho Digestivo, instalou, desde 1980, a Residência em Cirurgia do Aparelho Digestivo. Após dois anos de Cirurgia Geral, o jovem residente ingressa no programa de residência especializada em cirurgia digestiva durante mais dois anos, isto é, R3 e R4.

O programa visa a formar um cirurgião com elevado nível de conhecimento capaz de atender pacientes de grande complexidade clínica, bem como realizar operações em todos os setores do tubo digestivo, incluindo as de grande porte. Com o advento da cirurgia laparoscópica, houve necessidade de estabelecer um programa para completar a formação do cirurgião do aparel ho digestivo.

Desde a introdução da cirurgia laparoscópi ca no Hospital das Clínicas, em outubro de 1990, passamos a estruturar um programa para formação do cirurgião em laparoscopia do aparel ho digestivo.

Acreditamos que os cursos rápidos, organizados em fins de semanas com treinamento em caixas pretas e em animais de laboratórios, têm objetivos
Unidade de Cirurgia Laparoscópica no período de outubro 1990 a dezembro de 1998, com índice de conversão de $0,9 \%$ e sem mortalidade. A ausência de acidentes operatórios e de complicações verificadas na experiência adquirida nas operações realizadas por nosso grupo cirúrgico, dá consistente suporte à orientação assumida pela Disciplina.

Conclusão. Reforçam-se os princípios que foram adotados dentro da atividade em hospital universitário, que são: necessidade de formação de profissionais dentro de programa bem estruturado, ensejando-Ihes preparo educacional humanístico e técnico, em torno de projeto pedagógi co longo, mas com grande e sólido embasamento informativo e prático.

UnITERmos: Ensino médico. Cirurgia. Laparoscopia. Aparelho digestivo. Colecistectomia. quase que excl usivamente de gerar conhecimentos informativos teóri cos com demonstração da apareIhagem e dos procedimentos.

Em breves termos, esses cursos "informam", mas "não formam". Funcionam como o "test drive" das empresas concessi onárias de automóveis. E mbora com instrumentos diferentes, os obj etivos são semel hantes: na via comum final, a familiarização com o emprego rotineiro do equipamento só é conseguida depois que o consumidor o adquiriu.

\section{ROTEIRO DE TRABALHO}

\section{a) F unção do responsável pelo ensino}

Com todos esses recursos em mãos, cabe ao responsável pelo ensino de cirurgia do aparelho digestivo integrar, coor denar e estimular as ações, ensejando oportunidades a todos os interessados para atingir a meta de formar médicos de el evada qualificação profissional.

\section{b) Atividade e oportunidade acadêmica}

Estrutura da educação médica na Facul dade de 
Medicina da U niversidade de São Paulo - O acadêmico da nossa Faculdade de Medicina participa hoje de um programa curricular, formado substancialmente pelo currículo nuclear e optativo. Nas atividades extra-curriculares existem "Ligas" do Centro Acadêmi co Oswal do Cruz, que têm funções de aglutinar acadêmicos dos vários graus, identificados com os mesmos objetivos de formação e ou de aspirações futuras voltadas para o campo profissional. As "Ligas" estabelecem uma excelente via de dupla mão de comunicação e integração do Centro Acadêmico com as disciplinas dos vários departamentos na nossa F aculdade de Medicina.

$\mathrm{Na}$ estrutura de grande porte da Faculdade de Medicina da Universidade de São Paulo, os responsáveis pela educação médica detêm recursos materiais e humanos para formar médicos de grande qualificação profissional. Os recursos humanos são muito favoráveis, bilateralmente, em nível docente e discente.

O jovem acadêmico de medicina, antes do $4^{\circ}$. ano, entra na "Liga de Cirurgia Videolaparoscópica", do Centro Acadêmico Oswaldo Cruz. Nos espaços disponíveis da grade curricular passa a ter contato com método, com todo o seu instrumental, estuda a literatura concernente, anali sa as películas, coopera na realização de vídeos, assiste operações, participa de trabalhos científicos, de reuniões clínicas e científicas, bem como de congressos médicos como colaborador de trabalhos.

No $5^{\circ}$ ano de Graduação, como I nterno, ingressa no programa de aprendizado em serviço, na atividade hospitalar, acompanhando pacientes em regime ambulatorial e no pré, intra e pós-operatório.

Terminado o curso, durante a residência de $1^{\circ} \mathrm{e}$ $2^{\circ}$ ano, parti ci pa de intervenções com o emprego do método em Cirurgia Geral.

No $3^{\circ}$ e $4^{\circ}$ ano de resi dência especializada já atua na linha do programa com maior intensidade. Durante três meses, em tempo integral, participa das operações na U nidade de Cirurgia Laparoscópica. O objetivo é fazer o residente absorver o espírito de disciplina, dedicação e responsabilidade da equipe, familiarizando-se com o método, instrumentos e o meio. Rapidamente, vai desenvol vendo as suas aptidões técnicas, mas tendo como centro e objetivo maior o paciente, conhecendo bem seus aspectos clínicos e psicológicos, estreitando e aperfeiçoandose na relação médico-paciente e procurando sempre o resultado social do tratamento cirúrgico, objetivando melhor qualidade de vida para o paciente.

Com a incorporação dos princípios éticos e técnicos da sua formação, de imediato, vai entender o conceito que o importante é o seu compromisso com o paciente e a sua cura e não com o método.

Assim, de maneira progressiva e paulatina, sempre com a presença de pelo menos um membro sênior da equipe, vai assumindo atitudes, desde auxiliar, com sustentação de pinças de apresentação, depois da lente, e introdução de trocartes, iniciando as intervenções de maneira parcial até a sua realização compl eta.

N uma estrutura cada vez mais aperfei çoada, em relação aos recursos humanos e materiais, o residente de $4^{\circ}$ ano, num período de três meses de estági o na Unidade de Cirurgia Laparoscópica da Disciplina de Cirurgia do A parel ho Digestivo, chega a real izar cerca de 50 intervenções, 30 das quais são col ecistectomias Iaparoscópicas e as restantes consistem em laparoscopias diagnósticas para estadiamento de lesões, biópsias hepáticas, gastrostomias e jejunostomias vídeo-assistidas, além de participar como auxiliar em vários outras intervenções laparoscópicas avançadas, como procedimentos sobre a transição esôfago-gástrica, megaesôfago e hérnia hiatal, gastrectomias, esofagectomias, espl enectomias, enterectomias e col ectomias.

O modelo de formação e preparo do cirurgião do aparel ho digestivo que temos implantado com rigor, profundidade e seriedade, é certamente, responsável pelos resultados de nível de excelência obtidos.

\section{CASUÍSTICA}

Durante o período de outubro de 1990 até dezembro de 1998, for am real izadas 1.890 colecistectomias laparoscópicas na Disciplina de Cirurgia do ApareIho Digestivo, Unidade de Cirurgia Laparoscópica, Hospital das Clínicas da Universidade de São Paulo. O caso número 72 apresentou hematoma da cúpula hepática de causa desconhecida, de evolução incontrolável, chegando ao óbito depois de várias semanas. Os 1.818 casos operados, sucessivamente, fazem parte deste estudo. Destes, $11,1 \%$ eram doentes idosos, mai ores de 70 anos. Além disso, 45,6\% apresentavam doenças associadas, variando de ASA 1 a 4, como mostra o quadro a seguir:

\begin{tabular}{|lr|}
\hline Doenças Associadas & $\%$ \\
\hline Hipertensão arterial sistêmica & 27,7 \\
Cardiopatia & 8,3 \\
Obesidade & 5,6 \\
Pneumopatia & 2,4 \\
Cirrose hepática & 1,2 \\
HIV positivo & 0,3 \\
Coagulopatia & 0,1 \\
\hline
\end{tabular}

Do total, 95\% eram portadores de colecistite crônica calcul osa; 3,5\% de colecistite aguda; 0,9\% 


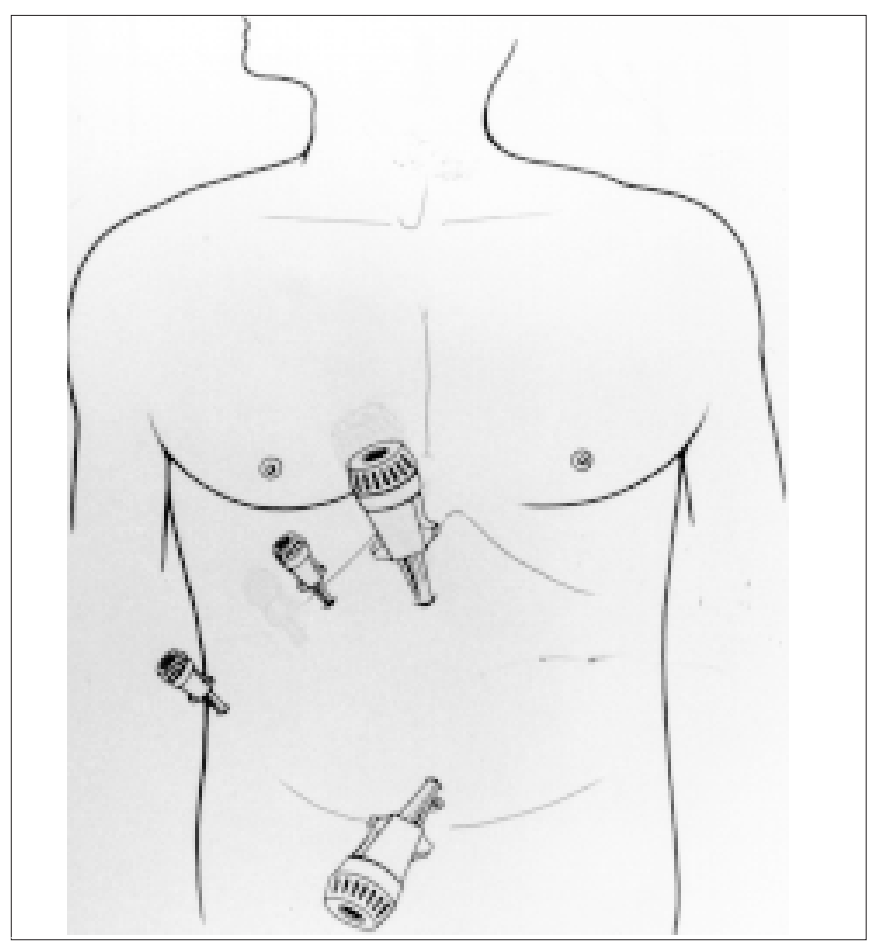

Fig. 1 - Posicionamento dos trocartes

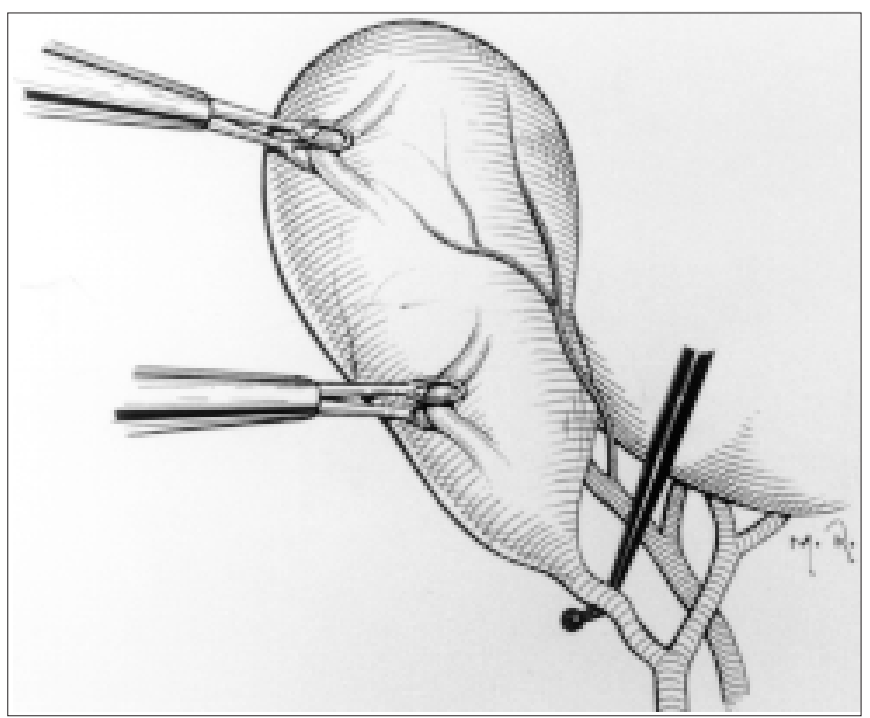

Fig. 2 - Modo de apresentação da vesícula biliar

de colecistite esclero-atrófica; $0,3 \%$ de pólipo de vesícula, 0,15\% de neoplasia de vesícula e 0,15\% de Síndrome de Mirizzi.

A colangiografia intra-operatória, considerada como prática de rotina, foi realizada em $90,6 \%$ dos pacientes. Com isso, foram diagnosticados $73 \mathrm{ca}-$ sos de col edocol itíase, dos quais 33 eram total mente insuspeitos.

Até 1998 houve 0,9\% de conversões, em conseqüência de causas diversas, como coledocolitíase, intolerância ao pneumoperitônio com alterações hemodinâmicas ou respiratórias.

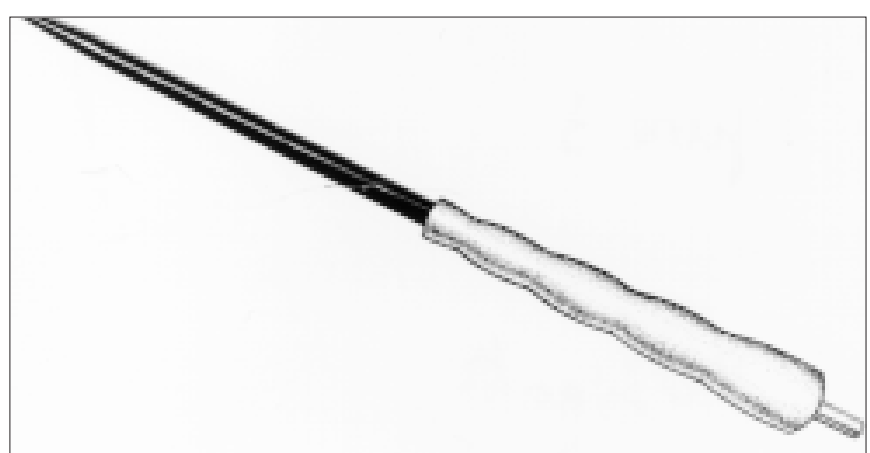

Fig. 3 - Instrumento para dissecção com extremidade esférica

\section{MÉ TODO}

A col ecistectomia laparoscópica foi sistematizada com vários passos que são rigorosamente seguidos a cada intervenção.

1) posição do paciente na mesa operatória - O paciente é colocado em decúbito dorsal horizontal sob anestesia geral. A monitorização per-operatória é realizada com cardioscopia, monitorização não-invasiva da pressão arterial, oximetria de pulso e capnografia ${ }^{1}$.

2) colocação de quatro trocartes - A posição de trocartes é feita segundo a Fig. 1.

$\mathrm{Na}$ região supra-umbilical, um de $10 \mathrm{~mm}$ para colocação da ótica e um de $5 \mathrm{~mm}$ no flanco direito para tração cranial da vesícula. Os outros dois trocartes de trabalho são colocados na mesma linha, um no hipocôndrio direito de $5 \mathrm{~mm}$ para apreensão da vesícula e outro no epigástrio, lado esquerdo do ligamento redondo, de $10 \mathrm{~mm}$, para dissecção e hemostasia.

A pós a abertura da pel e na região supra-umbilical, procede-se a dissecção da aponeurose, que é apreendida com pinças de Backaus, praticando-sesuspensão da parede abdominal. Esta manobra de elevação da parede abdominal facilita tanto a introdução da aguIha de Veress, como a realização do pneumoperitônio. Quando a pressão atinge $14 \mathrm{~m} \mathrm{mHg}$, acusada no painel de controle do insuflador, sempre com a parede abdominal firmemente suspensa, é, então, introduzido, cuidadosamente, o trocarte. Se houver qualquer eventualidade nesta fase que não permita essa seqüência ou quando houver intervenção cirúrgica prévia, é prudente realizar sutura em bol sa com prolene 0 e proceder a abertura cuidadosa da aponeurose e do peritônio, eatravés de manobras di gitais com a ponta do indicador voltada para o peritônio, abre-se um espaço para introdução do trocarte.

Os demais trocartes são colocados sob visão direta guiada pela ótica, preferentemente de 30 graus. A seguir o paciente é posicionado em proclive e decúbito lateral esquerdo. 


\begin{tabular}{|c|c|c|c|c|c|}
\hline & País & Casos & $\begin{array}{c}\text { Lesão de } \\
\text { via biliar } \\
\text { principal } \\
\%\end{array}$ & $\begin{array}{c}\text { Conversão } \\
\%\end{array}$ & $\begin{array}{c}\text { Mortalidade } \\
\%\end{array}$ \\
\hline $\begin{array}{l}\text { Cuschieri } \\
1991\end{array}$ & Europa & 1,236 & 0.33 & 3.6 & 0 \\
\hline $\begin{array}{l}\text { Baird } \\
\text { 1992(*) }\end{array}$ & $\begin{array}{c}\text { USA } \\
\text { (Atlanta) }\end{array}$ & 800 & - & 2.3 & 0.13 \\
\hline $\begin{array}{l}\text { Deziel } \\
1993\end{array}$ & $\begin{array}{c}\text { USA } \\
\text { (Chicago) }\end{array}$ & 77,604 & 0.6 & 1.2 & 0.04 \\
\hline Barkun & Canada & 1,300 & - & 6.2 & 0.08 \\
\hline \multicolumn{6}{|l|}{1993} \\
\hline $\begin{array}{l}\text { Gouma } \\
1994\end{array}$ & Holand & 2,932 & 1.09 & - & 0.07 \\
\hline $\begin{array}{l}\text { Steele } \\
1995\left(^{*}\right)\end{array}$ & $\begin{array}{c}\text { UK } \\
\text { (Nottinghan) }\end{array}$ & 650 & 4.3 & 19.0 & 0.15 \\
\hline $\begin{array}{l}\text { Richardson } \\
1996\left(^{*}\right)\end{array}$ & Scotland & 5,913 & 0.6 & 13.9 & 0 \\
\hline $\begin{array}{l}\text { Majeed et al. } \\
1996\end{array}$ & $\begin{array}{c}\text { UK } \\
\text { (Sheffield) }\end{array}$ & 100 & 1 & 20.0 & 工 \\
\hline Pinotti 1998 & $\begin{array}{c}\text { Brazil } \\
\text { (São Paulo) }\end{array}$ & 1,818 & 0 & 0.9 & 0 \\
\hline
\end{tabular}

A apresentação do campo cirúrgico é realizada com a vesícula tracionada para cima e para fora, em direção à cúpula diafragmática di rei ta e o hilo é exposto com a tração lateral, segurando-se o infundíbulo para a direita e para baixo.

A dissecção é realizada com um instrumento de extremidade esférica, que permite a dissecção romba, calibração e mobilização das estruturas, sendo esta função importante no sentido de minimizar a falta da terceira dimensão, e também coagulação.

A dissecção deve ser feita evitando-se o arrancamento e a divulsão, respeitando-se os planos de clivagem. A técnica consiste em abertura do peritônio com a di ssecção acompanhando o contorno externo da vesícula, desde o infundíbulo, o colo vesicular e o ducto cístico, evitando o eixo longitudinal do hilo hepático, bem como a parte do triângulo de Callot, conferindo, assim, boa segurança no trabal ho cirúrgi co. O ducto cístico é isolado, parcialmente seccionado e cateterizado, sempre que possível, para realização da col angiografia intra-operatória. E m seguida, após análise criteriosa da mesma, é fei ta ligadura e secção completa do ducto cístico; em seqüência, a artéria cística é reconheci da, isolada eligada com clips metálicos. A vesícula é, então, retirada do leito.

A retirada da vesícula do abdome, em casos de suspeita de neoplasia, deve ser feita dentro de saco plástico protetor.

\section{RESULTADOS}

Na série apresentada não houve nenhum caso de lesão vascular ou de via biliar principal. Os pacientes foram realimentados no mesmo dia da intervenção cirúrgica e receberam alta no primeiro dia de pós operatório, estando aptos para reintegração profissional e esportiva.

Um paciente teve lesão transfixante do ducto cístico, que fora irregularmente dissecado com pinça ti po Maryland, e teve que ser reoperado por via laparoscópica para correção, evoluindo sem inter corrências. U ma paci ente hígi da, sem fatores predisponentes, apresentou no $1^{\circ}$.PO quadro de tromboembolismo pulmonar extenso, necessitando de heparinização plena, porém recebeu alta após 10 dias.

\section{DISCUSSÃO}

Col ecistectomia laparoscópica tem si do utilizada como método de el ei ção no tratamento da col el itíase há aproximadamente 10 anos. Toda a experiência vivida nos diferentes centros possibilitou concluir que o método é seguro e benéfico, sem os incovenientes da dor incisional no pós-operatório, conferindo também realimentação e alta precoces, e restabel ecimento rápido das atividades habituais². 
A grande divulgação do procedimento e a troca de experiênci as têm feito com que os cirurgi ões vão progressivamente encontrando as justas indicações e aprimorando a metodologia operatória. Não obstante, a larga experiência mundial e a baixa mortalidade da intervenção, continuam sendo observados resultados desfavoráveis devidos à acidentes técnicos, como a introdução de trocartes provocando acidentes vasculares ou viscerais de graus variáveis, lesões de vasos da região hilar ou de ductos biliares que juntamente com as operações mal indicadas engrossam os índices de conversões para operações abertas ${ }^{3}$.

Problema sério, ainda, é a questão das lesões cirúrgicas das vias biliares, parciais ou totais, presentes em quase todas as casuísticas, verificadas também nos países de primeiro mundo, onde as condições de trabalho e de assistência são as mel hores ${ }^{4}$. Vários são os fatores intervenientes na sua origem, a falta de caracterização das estruturas, quer pela ausência da terceira dimensão ou pelas alterações anatômi cas em função dos processos flogísticos agudos ou crônicos, anomalias congêni tas ductai s e pela apli cação de termocautérios de maneira indevida, são apontados como os principais ${ }^{5}$. Enfermos que ingressam nas operações confiantes de se curarem com procedimento "mini invasivos", despertam delas com incisões grandes, drenos e curativos inundados de bile. Necessitam de intervenções repetitivas para tratar o acidente cirúrgico, aumentando os gastos e ônus sociais. Algumas lesões são tão graves, com conseqüências parenquimatosas secundárias, que já estamos assistindo as primeiras indicações de transplantes de fígado para estes doentes.

Nossa casuística, em comparação com outros grandes centros mundiais (Tabela 1), mostra ausência de aci dentes intra-operatórios e de mortalidade com baixos índices de conversão verificadas na experiência adquirida sobre 1818 sucessivas operações realizadas pelo nosso grupo cirúrgico; isso dá consistente suporte a orientação assumida desde outubro de 1990 a dezembro de 1998. Reforçam-se os princípios que temos adotado dentro da atividade em Hospital U niversitário, que são: a necessidade da formação de profissionais dentro de um programa bem estruturado, ensejando-se um preparo educacional humanístico e técnico em torno de um projeto pedagógi co longo, mas com grande e sól i do embasamento informativo e prático. O futuro do sucesso do cirurgião está em incorporar no seu patrimônio educativo a realização de um trabalho de rigor onde deverão prevalecer o planejamento, execução e condução de ato operatório bem siste- matizado com atitudes cuidadosas, lógicas coordenadas e sucessivas.

\section{SUMMARY}

Digestive tract laparoscopic surgeon formations - experience in $\mathbf{1 8 1 8}$ laparoscopic choleystectomies without complications or mortality

Since the beginning or Iaparoscopic surgery on University of Sao Paulo Medical School Clinics Hospital, the Digestive Surgery Division established an educational program for surgeons of the alimentary tract.

PuRPose. The course structure includes the information on medical school, extension in Iaparoscopic surgery league, and surgical formation during the residence, mainly in the fourth year, with a three months period in the Laparoscopic Surgery Unit.

METHOD. This model of surgical formation is certainly responsible for the excel lent results obtained.

RESULTS. An example is the performing of 1818 cholecystectomies in the Laparoscopic Surgery Unit from 1990 till 1998, with 0.9\% convertion, and no mortality. The absence of operative accidents or complications give support to the orientation assumed in our Division.

ConClusion. In a university hospital there is the necessity of professional formation in a well structured program, with humanistic and technical education, in a large period of rotation, but with a solid information and practical structure. [Rev Ass Med Bras 1999; 45(4): 337-41]

KEY WORDS: Medical education. Surgery. Laparoscopy. Alimentary tract. Cholecystectomy.

\section{REFERÊNCIAS BIBLIOGRÁFICAS}

1. Klajner, S. - O valor da capnografia e oximetria de pulso na monitorização em cirurgia vi deol aparoscópica. Dissertação de Mestrado apresentada em 1998.

2. Libanori, H.T. - Estudo comparativo da resposta ao traumatismo cirúrgi co em pacientes submeti dos a col ecistectomia por laparoscopia e laparotomia. Tese de Doutoramento apresentada em 1996.

3. Deziel, D.J .; Millikan, K.W.; E conomou, S.G. et al. - Complications of laparoscopic cholecystectomy : a national survey of 4,292 hospitals and na analysis of 77,604 cases. Am.J .Surg. 165: 9-14,1993.

5. Rocha, P.R.S.; Ferreira, J .T.; Diniz, M.T.C. - Resultados da colecistectomia laparoscópica no Brasil : análise de 10.044 casos. In: Paula, A.L.; Hashiba, K.; Bafutto, M. - ed. Cirurgia vídeo-laparoscópica. Goiânia, Gráfica e E ditora Bandeirante Ltda., 201-211, 1994.

6. Santo, M.A. - Colecistectomia videolaparoscópica. Análise de aspectos clínicos efuncionais da suspensão mecânica da parede abdominal. Dissertação de M estrado apresentada em 1995. 